\title{
Smart Grid Communication Infrastructure, Automation Technologies and Recent Trends
}

\author{
Masood Aslam*, Nadeem Shahbaz, Rauf Ur Rahim, Muhammad Gufran Khan \\ Electrical Department, National University of Computer \& Emerging Sciences, Islamabad, Pakistan \\ Email address: \\ f169101@nu.edu.pk (M. Aslam), 169104@nu.edu.pk (N. Shahbaz),f169039@nu.edu.pk (R. Ur Rahim), m.gufran@nu.edu.pk (M. G. Khan) \\ ${ }^{*}$ Corresponding author
}

\section{To cite this article:}

Masood Aslam, Nadeem Shahbaz, Rauf Ur Rahim, Muhammad Gufran Khan. Smart Grid Communication Infrastructure, Automation Technologies and Recent Trends. American Journal of Electrical Power and Energy Systems. Vol. 7, No. 3, 2018, pp. $25-32$.

doi: $10.11648 /$ j.epes. 20180703.11

Received: May 31, 2018; Accepted: June 12, 2018; Published: July 5, 2018

\begin{abstract}
In the last century, there was no major alteration in the structure of power grids. Studies have shown that existing infrastructure is no longer suitable to meet requirements of the future. To overcome the challenges and fulfil future requirements, we need a network that is reliable, efficient and intelligent. This has led to the development of emerging concept of smart grid (SG). Smart grids are the next generation of electrical power grids; a promising solution to overcome energy crises. Smart grid system is the integration of advanced computing and communication technologies with power system infrastructure. In this paper, we provide a survey of the communication infrastructure in smart grids that includes communication architecture and different network compositions, technologies, and intelligent functions that are employed in this architecture. This survey is expected to provide improved understanding of the technologies, its potential benefits and impact. Moreover, we intend to provoke interest amongst the research community to discover more about this emerging area to realize the potential of smart grid concept.
\end{abstract}

Keywords: Communication Technologies, Smart Grid, AI, Smart Meter

\section{Introduction}

The power grid infrastructure being used has been unchanged for about 100 years. This power grid did well in providing power supply to the consumers until last 2 decades. In the past decades, there have been many blackouts as well and they have caused a lot of inconvenience to the consumers [1]. As countries are becoming advanced technologically electricity consumption may reach at a point where it will be difficult to manage it if it is left unattended. Demand for the electricity is also increasing day by day and present power grid is not sound enough to counter the demands. Hence this power grid is not suited to meet the requirements of the $21 \mathrm{st}$ century [2]. There are many reasons which include old and worn out components, climate change, exhaustion of energy resources, one-way communication, and constant failure of equipment [2]. We need a modern and dynamic infrastructure to cope with all these challenges.

To overcome all these challenges an intelligent power grid is the need of the hour. Thus, the concept of smart grid has emerged. The smart grid has improved reliability, control and safety [3]. A smart grid is a combination of various automatic transmission and distribution systems operating in a reliable and efficient way. It has a two-way communication infrastructure linking components through sensor nodes and allows communication between the components. The components used in home and generation unit use online system to communicate with each other in real time. Equipment failure and natural catastrophes that are the main causes of blackouts can be prevented through this online system by continuous monitoring.

Apart from efficient energy management, smart grids can also prove to be environment friendly. Most of the power stations use fossil fuels for electricity generation. Moreover, a lot of electric power is wasted during distribution [4]. The electricity generated has low efficiency and it is not good for the environment as it causes pollution. Current power grids emit a lot of carbon dioxide, and we are wasting already limited natural resources [5]. Alternatively, renewable energy resources are becoming popular because these are carbon- 
free and good for the environment. Communication technologies will help in integration of renewable energy with smart grid and to save energy when the demand is low.

In order to make smart grids adaptive and intelligent to varying system requirement and load conditions, Machine Learning (ML) methods can be applied to provide solution to the complex problems. This data-driven approach includes algorithms like Artificial Neural Networks (ANN), fuzzy logic, Support Vector Machine (SVM) etc. These will help in making applications that can adapt and address any problem that arises which mill make the grid more reliable.

Still solutions to many technical challenges of smart grids are pending. The main objective of this paper is to provide a survey on smart gird communication architecture and communication technologies in smart grid. Some these wired and wireless technologies are DSL, fiber optic, PLC, ZigBee, WLAN, WiMAX, cellular and satellite. The communication architecture includes WAN, LAN, NAN, BAN etc. The study of communication architecture of smart grids is still at the primeval stage. Our survey might not have all the relative information discussed, but it delivers a primitive summary of the smart grid research and future considerations. Secondly, the paper presents recent trends in the modern grid implementation such as Artificial Intelligence (AI), Cloud Computing, Smart metering and Internet-of-Things (IoT).

The survey is organized as follows. Section II presents background and related work. Section III contains smart grid communication network architecture. Section IV includes networking technologies that are currently used in smart grids. Section V presents the latest trends for automation of smart grids. Finally, Section VI presents conclusions.

\section{Background and Related Work}

The communication architecture of smart grids has become a very important topic these days because of the increasing research on smart grids. It is very important in smart grid communication network to choose an effective communication topology [6]. In the literature, most studied issue is the communication network technologies and its network requirements [6]. Smart grid technology goes back to 1980 when the first advanced automatic meter was used to show electricity consumption at different times of the day. In 2000 smart meter was deployed in Italy using Power line communication (PLC) [6]. PLC is used to transmit alternating current (AC) and data simultaneously. At that time data rates were very low usually up to $(100 \mathrm{kbps})$ and frequency was also low $(3-500 \mathrm{KHz})$. So repeaters were used to increase the coverage area [6]; this technology was used till 2010. In [7], the role of PLC in smart grids was discussed; authors suggest that PLC will prove to be a better technology for wired communication.

In 2011, along with PLC which was a wired technology, wireless technologies such as Home Area Network (HAN) and Wireless Area Network (WAN) started to become popular. The main role of HAN in smart grids is to integrate meters with smart grid [8]. WAN, on the other hand, is used for wide-area protection, control and monitoring in the smart grid [8]. In [9], author has presented integration of PLC and wireless technologies with smart grid. By 2011, the PLC data rate increased to $100 \mathrm{Mbps}$ and the frequency to $5000 \mathrm{KHz}$, so coverage area increased as well. Moreover, a short range wireless technology called Zig-bee along with HAN was used to control home appliances automatically [9]. WAN technology was still developing at that time. The idea was to use Wi-Max for machine-to-machine communication, but Wi-Max required a licensed spectrum and was very costly. Wi-max was also not deployed at large scale so there were some risks involved as well.

In [10], authors presented the idea of communication of smart grids with electric vehicles. IP based network using $3 \mathrm{G}$ in smart grid also developed; this technology was suggested to be used in integration of meters with generation units. In some of the papers related to communication in smart grid i.e. [4] and [11], authors presented renewable energy resources and their integration with communication architecture. Authors in [12] presented a survey of latency and bandwidth requirements of network applications in transmission.

Later, there was a lot of interest and focus on wireless communication technologies such as Bluetooth, WiFi [13] after 2013. Neighborhood Area Network (NAN) also gained popularity; NAN is used to link all users of regional area, which makes it the most important part of the smart grid. It connects the gap between HAN and WAN. As use of communication technology in smart grids was increasing so was the threat of security of using these technologies. So, in 2013 most research was on quality of service, traffic requirements and different threats such as privacy and security of users [14]. Some of the papers such as [5] provide a review of cyber security issues in communication architecture. Reference [15] presented a review about traffic requirements and characteristics of communication networks in smart grids. Moreover, [16] presented communication technology assessment and its different requirements.

The concept of network virtualization in smart grids was presented in [17]. Network virtualization means combination of hardware and software network into a single entity, called a virtual network. So network virtualization in smart grid is mapped PLC and wireless technologies simultaneously. This allows different subcarriers to different NV's. Simultaneous transmission in several subcarriers helps in improvement of transmission. Some studies have emphasis on specific communication technologies i.e. HAN [8], NAN [18].

During 2015 and 2016, most of the research work is done on improving Neighborhood Area Network (NAN) as it is the most crucial part of a smart grid. The concept of LTE integration with NAN is given by [19] in which Device-todevice (D2D) communications is used with LTE. This can reduce delay and boost reliability, but it is still too early because LTE cannot support all the advanced NAN technologies. 


\section{Communication Network Architecture}

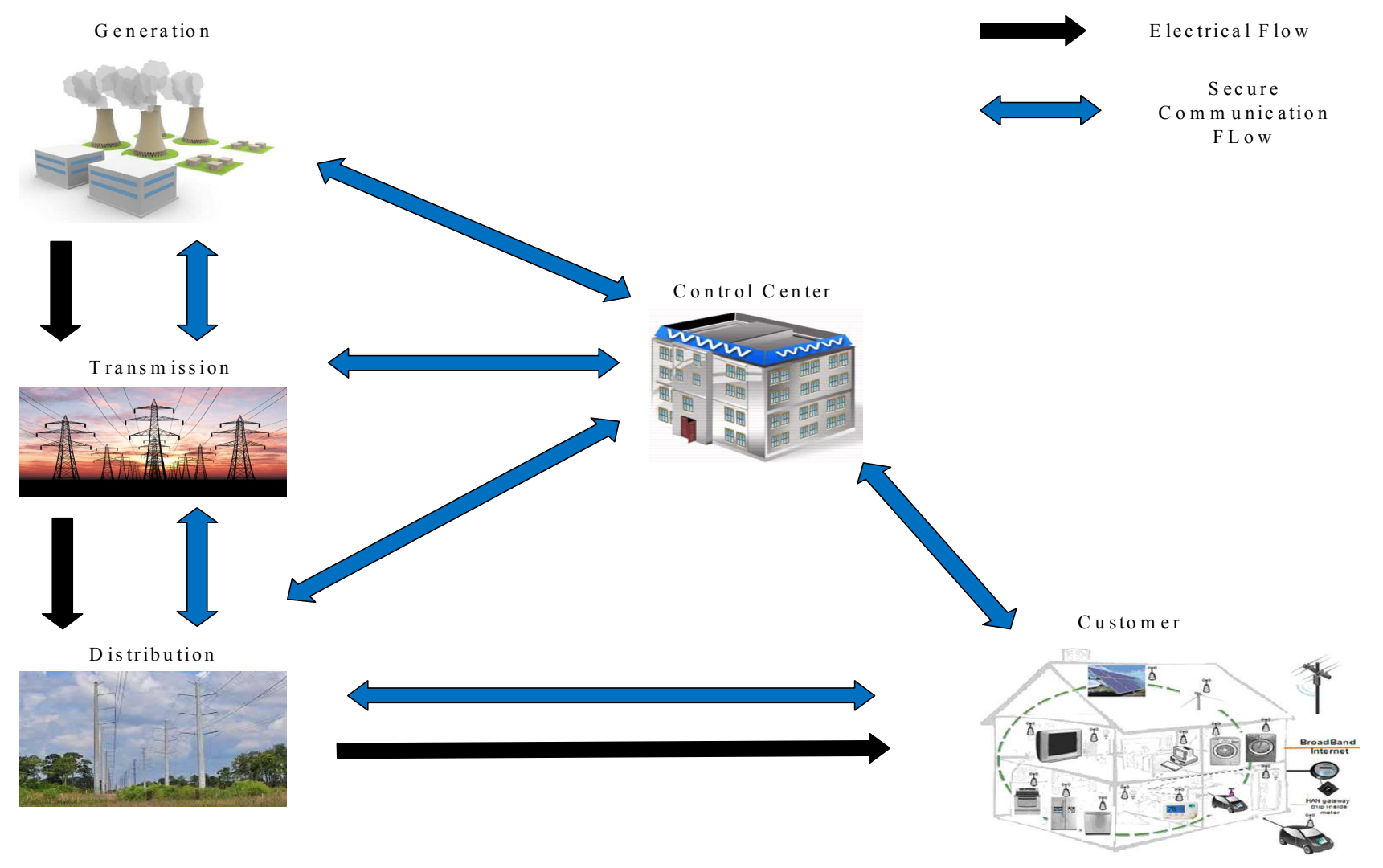

Figure 1. Communication Network Architecture.

Figure 1 shows communication architecture of smart grid which includes neighborhood area networks (NANs), home area networks (HANs), wide area networks (WANs), substations and data centers [20]. These networks are briefly described in the following subsections.

\subsection{Wide Area Network (WAN)}

WAN is a combination of two networks i.e. core and backhaul network. Core network provides connectivity between utility and substation systems and backhaul connects the NAN network with core network [21]. This can help to prevent power outages and blackouts by providing real-time information of the power grid [13]. It serves as a backbone for small area networks that aids power grid at various locations [6]. It supports real-time control, protection and monitoring applications. Protection application offers automatic protection system to prevent widespread blackouts and unexpected events. This system is useful when dealing with unexpected contingencies as it has short amount of reaction time to avoid blackouts and failures [13]. Control application gives the capability of self-healing to smart grid. This application helps in performing quick operation of generator tripping and provides voltage support to large power systems. This application also acts as an extra layer for prevention of blackouts. WAN monitoring application provides real-time data for Intelligent Electronic Devices (IEDs) and Phasor Measurement Units (PMUs). So, monitoring application provides an opportunity to the power grid to improve voltage stability and also helps in detecting frequency oscillations or instability [22].

\subsection{Home Area Network (HAN)}

Home Area networks can be classified as part of network architecture on customer side. HAN's are usually used in residential and business consumers with an electric utility to enable communication between them [11]. Building Area Network (BAN) is also a part of HAN and is considered more complex than HAN. It is a combination of connected devices, management software and dedicated local area networks. BAN enables industrial or commercial customers to connect with an electric utility [23]. HAN supports communication between the smart meter and appliances that are being used in home, industry or building. It also supports other several services that include demand response, prepaid, real-time pricing and load control [19].

Main requirements for communication in HAN are low cost, less power consumption and most importantly secure communication. Some of the technologies that are introduced in HAN are ZigBee, WLAN, and PLC.

\subsection{Neighborhood Area Network (NAN)}

NAN is the best described as a bridge between WAN and HAN. NAN's are used to collect data from nearby points with the help of Intelligent Electronic Devices (IEDs) that are 
widely deployed in whole area [18]. It is a two-way advanced communication technology that gives information of electricity usage and system control to smart meters. As compared to WAN the data rate is not that high, and also the transmission power is low for transmission in short range [18].

Wireless communication technologies such as low bandwidth channel are usually used in NAN's [24]. Advanced metering infrastructure (AMI) is deployed by NAN and the range of applications is expanding rapidly i.e. integration of distributed energy resources and advanced distributed automation [25]. Two types of applications are used in NAN i.e. field based and customer based applications. Field based include sensors, transmission lines and voltage regulators; and customer based application is related to end customers. Customer based applications need to be highly scalable and timing sensitivity is not a big issue while field based applications are time sensitive in nature [6]. Therefore, both applications have a choice to share each other's networks. This shared network will help in minimizing development cost whereas the dedicated network will give additional security and real-time communication capability [6].

Number of users joining smart grid is increasing day by day and it is causing delay as the packets generated by Electrical Devices have to pass through many interconnected networks. So to overcome this we need a solution in which WAN is combined with efficient routing strategy. NAN's data rate and coverage area requirements depend on applications e.g. for smart meter data rate is more than $100 \mathrm{kbps}$ which depends on data size of meters and number of meters. WLAN, PLC and ZigBee are some of the technologies on which NAN can be implemented.

\section{Network Technologies}

All the technologies that are used in communication network infrastructure of smart grids are presented below.

\subsection{Power Line Communications (PLCs)}

PLC, we can say is a 'no new wire' technology [26]. A bidirectional technique for communication between consumers and the power grid is the requirement of smart grid communication infrastructure to enhance the safety, reliability and efficiency of the transmission system [27]. PLC has proved to be a possible alternative for this, from home appliances control to distribution unit based management and control, and that too with already available network.

This is a suitable technique for street light control. PLC can be used in rural areas very efficiently as there are no other communication infrastructures present. The main advantage of PLC as compared to wireless technologies is that it cannot be tempered as it is powered by $230 \mathrm{~V}$ which gives security against any kind of tempering. Some of the disadvantages of using PLC are signal distortion, noise and interference.

In 2004, HomePlug Standards solved the issue of distortion in PLC. So far, NB-PLC provides highest data rate for applications related to smart grid. It gives $46.5 \mathrm{kbps}$ for distance of $500 \mathrm{~m}$ and it is being applied in Taiwan for broadcasting control of intelligent air conditioners. In Europe, PLC is providing better coverage as compared to the $\mathrm{Wi}-\mathrm{Fi}$ because it is harder for $\mathrm{Wi}-\mathrm{Fi}$ to penetrate those housing/buildings.

\subsection{Fiber Optic}

Fiber optic is the essential part of the communication technologies. Fiber optic not only supports smart grid applications but both wired and wireless applications as well. The communication infrastructure requires significant fiber optic transport capacity, whether it is wired or wireless infrastructure [28]. Due to high speed, optical fiber is considered as the best transmission medium for SG applications. For single wavelength transmission, optical fiber provides data rate up to $10 \mathrm{Gbps}$ and for wavelength division multiplexing data rates from $40-1600 \mathrm{Gpbs}$ can be achieved [26]. Only disadvantage is that the installation and monitoring of fiber optic is quite expensive.

There are several forms of fiber optics communications which include Passive Optical Network (PON), Synchronous Optical Networking (SONET)/Synchronous Digital Hierarchy and Wavelength Division Multiplexing (WDM). Depending on application, the form of optical fiber is selected on the basis of response time and quality of service.

a) PON is a point-to-multipoint network architecture, which utilizes optical splitters to enable a single optical fiber to serve multiple customers.

b) WDM is an effective technology to exploit bandwidth capacity available in optical fiber. Multiple wavelengths are used to carry several data streams simultaneously over the same fiber in WDM networks.

c) SONET/SDH is a time-division multiplexing (TDM) architecture that was designed to carry high capacity traffic [16].

\subsection{Digital Subscriber Line (DSL)/Broadband Communications}

DSL is used on ordinary telephone lines for transmission of digital data [13]. The signals transmitted by this technology are of more frequency than a dial-up connection [29] which allows larger bandwidth and faster speeds. There are three types of DSL that are being used which include Asymmetric Digital Subscriber Line (ADSL) and Symmetrical Digital Subscriber Line (SDSL). Currently ADSL is the popular DSL communication as it offers much higher download speed; in SDSL upload and download speeds are almost equal.

DSL is appropriate for providing data collection services between an electric utility and homes [13]. The DSL service is already available to almost all users on their existing telephone lines. So, it can be used for smart grid applications such as smart meters. DSL has an advantage that if there is any outage in communication link, service provider is going to be 
responsible for this; and it is said to be very reliable because outages on DSL are few and far between. DSL has many disadvantages as well. Its efficiency decreases with increase in distance from service provider. In case of any outage, restoration time will be much longer; therefore, we can say that DSL is not suitable for critical applications in SG.

\subsection{ZigBee}

It is a wireless network based on the standard IEEE 802.15.4 [30]. It is very effective and cost saving solution. ZigBee operates on ISM (industrial, scientific and medical) bands i.e. unlicensed frequency of $2.4 \mathrm{GHz}$ worldwide. It can cover area up to $30-40 \mathrm{~m}$ indoor and unlimited area while using a mesh network. It is mostly used for energy monitoring and home/building automation. ZigBee is although a mesh network but it also supports other topologies that are star and tree. ZigBee uses 128-Bit Advanced Encryption Standard (AES) or Access Control List to provide security. A mesh network is de-centralized and all nodes can manage themselves and can dynamically route and connect to new nodes if needed. An association of firms known as ZigBee Alliance has offered different type of standards which can be used for a number of applications i.e. home automation and meter reading, energy monitoring and smart lighting. ZigBee is ideal for smart grid applications because of its robustness, mobility and low deployment cost.

ZigBee has some disadvantages as well. Memory size is too small, delay requirements are also less and main problem is its interference with appliances that share the transmission medium same as ZigBee. This interference can result in communication channel corruption. So, to make ZigBee viable for smart grid applications in long run there is a need to develop schemes which can overcome all these problems.

\section{5. $W L A N$}

WLAN is a wireless high speed network and internet communication technology commonly known as Wi-Fi. Based on 802.11 IEEE series standard and includes 802.11, $802.11 \mathrm{a}, 802.11 \mathrm{~b}, 802.11 \mathrm{~g}$ and $802.11 \mathrm{n}$ standards as well. 802.11 is famous standard used in LAN and it gives data rate of $150 \mathrm{Mbps} \max$ and $250 \mathrm{~m}$ of coverage distance. Other standards $802.11 \mathrm{a}, 802.11 \mathrm{~b}, 802.11 \mathrm{~g}$ and $802.11 \mathrm{n}$ give data rates of 54, 11, 54 and $300 \mathrm{Mbps}$, respectively. WLAN operates on different frequencies i.e. $2.4 \mathrm{GHz}, 3.6 \mathrm{GHz}$ and 5 GHz. It can also be considered as a substitute for NAN's and HAN's. WLAN is capable to provide transmission from point to point and point to multipoint. Security issues are addressed in WPA-2 IEEE standard 802.11i that uses Advanced Encryption Standard [30].

All the electronic devices use Wi-Fi whether it is mobile phone, gaming console or laptop. WLAN uses IP protocol to communicate over the network with other devices. One issue with Wi-Fi is that it has limited number of channels so only a limited number of devices can be connected at a time [30]. It is more useful for applications which can work in interference and have requirements of smaller data. WLAN can intelligently monitor and control communication at substations by using sensors and wireless interfaces. For reliability of substations optical fiber can be used with wireless links. WLAN along with mesh network can be used to develop a dynamic network that is self-healing and selforganizing.

\subsection{WiMAX}

WiMAX is a 802.16 IEEE standard and operates at frequency bands of $2.3 \mathrm{GHz}, 2.5 \mathrm{GHz}, 3.3 \mathrm{GHz}$ and $3.5 \mathrm{GHz}$ and an unlicensed frequency of $5.8 \mathrm{GHz}$. It is a $4 \mathrm{G}$ technology and it can be characterized by security protocols (AAA, AES etc), low latency i.e. (10-50 ms), coverage area is up to $50 \mathrm{~km}$ with data rates up to $75 \mathrm{Mbps}$, low operating and deployment cost [16].

Some other standards used by WiMAX are IEEE 802.162004, 802.16e. The standards of WiMAX support two-way real-time communication between nodes. So, WiMAX can be upright contender for applications used in smart grids. These applications can be used for smart metering, distribution processes and monitoring of transmission.

WiMAX technology is suitable for Wireless Automatic Meter Reading (WAMR) as part of a utility's AMI network as it offers efficient coverage and high data rates. Secondly, WiMAX based AMI can easily be employed for provision of Real Time Pricing (RTP) signals to the consumers based on their real- time energy usage. In addition, using bi-directional WiMAX links, outages can be quickly detected and power restored resulting in increased reliability of power supply. Finally, sensor data can be transmitted over WiMAX links for monitoring purposes [30].

WiMAX also has some disadvantages. This technology cannot be easily implemented in urban areas because frequencies greater than $10 \mathrm{GHz}$ can't pass through buildings or we can say obstacles. Therefore, we need low frequencies as these frequencies can pass obstacles easily. But there is another problem as well that low frequency bands are already licensed so it may be required to lease them from third parties [13]. Bad weather can also affect the performance of WiMAX.

\subsection{Cellular Network}

Cellular network has been in use for last two decades. Although in the beginning it was only used for sending and receiving calls but, now a days, it is also used for widespread range of data services. It can be used for applications of smart grid because it can save additional time and building cost for designing a dedicated infrastructure. Cellular network is divided into different generations such as $2 \mathrm{G}$ and $3 \mathrm{G}$, and it operates on frequencies bands. Some of the features of current cellular networks are robust security, widespread coverage, low maintenance cost and higher data rates.

Long Term Evolution (LTE) or $4 \mathrm{G}$ is the latest development in the field of wireless communications. $4 \mathrm{G}$ provides the best data rates; it gives download speed of 300 
Mbps and upload speed of 75.4 Mbps. LTE has produced many new technologies as compared to its previous generations. One of the good feature of $4 \mathrm{G}$ is that it is compatible with its legacy standards which include CDMA 2000, UMTS, GSM/EDGE. Currently the version of LTE that is in use is LTE Advanced. It offers downloading speed up to 3.3 Gbps. LTE can have two key applications in smart grids i.e. controlling and automation of distribution system and smart meters [30].

\subsection{Satellite Communication}

Satellite communication technology transfers signals between two nodes also known as space communication. In this communication, the signal is sent to the satellite, the received signal is amplified and sent back to earth [30]. It is used for applications such as radio and television broadcasting, communication to planes, ships, vehicles and handheld devices. This technology offers microwave radio relay communication. By using satellite communication, it is possible to provide coverage to every part of earth. This is one of the reasons that it is used for communication in remote areas where it is not viable for other technologies [6]. Generally, this technology provides up to $1 \mathrm{Mbps}$ of data rate. This can be used for remote monitoring of distribution and generation units. This is the only viable method to use where wired and other wireless communication technologies are available. Satellite communication has so many disadvantages. Its cost of operation is very high and also buying or renting a satellite on space is not viable. Signals will take long time as they have to complete a round trip form earth to space and again back to earth [13]. Weather will also affect the signal quality in satellite communication.

\section{Smart Grid Automation}

All the technologies that are used in communication network infrastructure of smart grids are presented below. Electrical Power Systems or Power Grid are considered as the greatest artificial systems in the world with respect to area covered. Yet, these systems are weak as compared to other infrastructures and these systems can collapse easily in case of natural disasters. The blackouts that occur in result of these disturbances can cause serious damage to the grid and customer as well. AI, DR, Cloud computing and Smart Metering and IoT can play a big role in preparing for these natural disasters.

\subsection{Artificial Intelligence (AI) Algorithms}

Artificial Intelligence (AI) has slowly become the most important part of our life. It is used in most of our daily works. Using machines not only gives accurate results but also help in doing the work speedily. This technology is slowly being introduced to all the sectors to get accurate results and to reduce human work. Smart grid can also benefit from AI and machine learning algorithms. Computer technologies are not sufficient to provide solution of all the problems, so there comes the use of AI which is a powerful solution to these problems. AI can provide improvements in reliability, safety, cybersecurity of the systems, optimization and prediction.

With the integration of technologies, huge amount of data is transferred at different points that open up opportunities for security violations at different sectors. AI will prove very helpful in detecting the anomaly at any point by using different algorithms. Anomaly detection can play a key role in solving intrusions, as while detecting anomalies, disturbance of normal behavior indicate a presence of planned or unplanned induced attacks, defects, faults, and so on. The speed of anomaly detection is enhanced by using Machine Learning.

Another area where AI can play an important role is cloud computing. Collection of data in big volume then analyzing and storing the data in cloud environment. There will be continuous data generated by sensors, IED's etc. so an infrastructure should be in place to control all these sensors or devices and gather all the data generated by them. So we need adaptive algorithms and AI mechanisms to provide that infrastructure and allow distributed data management.

Currently different software architectures are employed but among them, the software, which has its origins in, distributed AI management Multi-Agent System (MAS). This system has the ability to provide flexible distributed systems, which makes them suitable for smart grid as smart grid, also support distributed system. So far it is believed that AI will play a key role in the life of electricity consumers and the future of smart grid but different in each case. AI will provide the algorithms that will support the whole infrastructure of smart grid. This will also help in providing best potentialities that smart grid has to offer.

\subsection{Demand Response (DR)}

Demand response is one of the most crucial part of smart grid. According to US Department of Energy DR is "a tariff or program established to motivate changes in electric use by end-use customers, in response to changes in the price of electricity over time, or to give incentive payments designed to induce lower electricity use at time of high market prices or when grid reliability if jeopardized"[13]. Therefore, from the definition we can understand that the idea is not only make DR eye-catching to its consumers to manage their daily power usage that will be beneficial for not only consumers but for the power grid as well.

The traditional DR mechanisms that are in use do not have the ability to make decisions at run time. They are limited to information of energy consumption received through patterns of daily usage. Due to lack of communication between consumer and utility makes it difficult to improve the energy efficiency of the power grid. So, with traditional DR mechanisms the utilities on try to match the supply which is very costly and impractical. Smart grid has the ability to interact in real-time with its customers so it requires efficient AI algorithms [31]. 
Another important feature that smart grid will have is that consumers will also be able to give energy back to the utility. This will help users and utility to use the energy efficiently and keep the demand response of electricity in check and also reduce the harmful emissions that are produced during electricity production.

\subsection{Cloud Computing}

Cloud computing is using resources over the network. This feature is necessary for smart grid. The amount data that is transferred in smart grid is very huge and it is not a good idea to use server or storage devices for this. The data has to transfer from consumers to electricity utilities. This will require many nodes for the communication and also their might be latency because of large amount of data. To overcome this, the best option is to use cloud computing. The data is transferred from one point to another in real-time. This process is also affordable and scalable.

Many of the technologies that are integrated with smart grid will get benefit from this technology. Demand Response as explained earlier will use cloud computing. Smart meters is another example.

Cloud computing has three types of services

a) Platform as a Service (PaaS)

b) Software as a Service (SaaS)

c) Infrastructure as a Service (IaaS)

All of these services are responsible for working of a cloud-networking infrastructure and provide different type of services.

\subsection{Smart Meters and IoT}

Many networking techniques discussed earlier need smart meter to perform their required operation. We can say that for two-way communication in smart grid, smart meter plays a vital role. It gets the information of the energy usage and then sends it to utility for billing. It stores realtime consumption of electricity used and the users can alter their electricity usage with the help of smart meter. Smart meters will have the ability to disconnect or connect a certain load and also monitoring of devices remotely in future. Customers will also get all the information if there is any power outage cause of outage, estimated restoration time, and public safety notice.

Smart meters and IoT technology have lot of benefits for the consumers as well as the utility. In case of any problem like if the utility needs to cut the electricity of a certain building or house, they can simply disconnect that building from the system remotely and when the issue is resolved the building can be connected. It has monetary benefits as well since utility personal does not have to travel to the specific location, they can simply do all the tasks remotely.

\section{Conclusion}

In this paper, we presented a survey on communication technologies and recent trends for automation of smart grids.
Our survey reviews the present research on communication networks. We have compiled different communication technologies and networks that can be used in smart grid communication infrastructure on customer side and distribution units. We have given advantages and disadvantages of all the smart grid communication technologies that can help utility engineers in making the right choice of communication technology when they design network for smart grid and its associated applications. In addition, some of the challenges of smart grids and recent trends such as AI, cloud computing and IoT have been discussed. We expect that presented work will provide better understanding of network requirements for variety of applications used in smart grids. A lot of research is still required for the smart gird before the communication infrastructure can be applied in smart grid for intelligent energy management.

\section{References}

[1] The Smart Grid: An Introduction, 2012. [Online]. Available: http://energy.gov/sites/prod/files/oeprod/DocumentsandMedia/ DOE_SG_Book_Single_Pages(1).pdf [Accessed: 23-April2017]

[2] Gungor VC, Sahin D, Kocak T, Ergut S, Buccella C, Cecati C, Hancke GP. Smart grid technologies: Communication technologies and standards. IEEE transactions on Industrial informatics. 2011 Nov; 7 (4):529-39.

[3] Gungor VC, Lu B, Hancke GP. Opportunities and challenges of wireless sensor networks in smart grid. IEEE transactions on industrial electronics. 2010 Oct; 57 (10):3557-64.

[4] Chen K-C, Yeh P-C, Hsieh H-Y and Chang S-C. Communication infrastructure of smart grid, 4th International Symposium on Communications, Control and Signal Processing, Cyprus, 2010, (pp. 1-4).

[5] Ma R, Chen HH, Huang YR, Meng W. Smart grid communication: Its challenges and opportunities. IEEE transactions on Smart Grid. 2013 Mar; 4 (1):36-46.

[6] Zhang Y, Sun W, Wang L, Wang H, Green RC, Alam M. A multi-level communication architecture of smart grid based on congestion aware wireless mesh network. In North American Power Symposium (NAPS), 20112011 Aug 4 (pp. 1-6). IEEE.

[7] Galli S, Scaglione A, Wang Z. Power line communications and the smart grid. In Smart Grid Communications (Smart Grid Comm), 2010 First IEEE International Conference on 2010 Oct 4 (pp. 303-308). IEEE.

[8] Namboodiri V, Aravinthan V, Mohapatra SN, Karimi B, Jewell W. Toward a secure wireless-based home area network for metering in smart grids. IEEE Systems Journal. 2014 Jun; 8 (2):509-20.

[9] Yu FR, Zhang P, Xiao W, Choudhury P. Communication systems for grid integration of renewable energy resources. IEEE network. 2011 Sep; 25 (5).

[10] Su H, Qiu M, Wang H. Secure wireless communication system for smart grid with rechargeable electric vehicles. IEEE Communications Magazine. 2012 Aug; 50(8). 
[11] Yan Y, Qian Y, Sharif H, Tipper D. A survey on smart grid communication infrastructures: Motivations, requirements and challenges. IEEE communications surveys \& tutorials. 2013 Mar; 15 (1):5-20.

[12] Kansal P, Bose A. Bandwidth and latency requirements for smart transmission grid applications. IEEE Transactions on Smart Grid. 2012 Sep; 3(3):1344-52.

[13] Pipattanasomporn M, Kuzlu M, Rahman S. Demand response implementation in a home area network: A conceptual hardware architecture. InInnovative Smart Grid Technologies (ISGT), 2012 IEEE PES 2012 Jan 16 (pp. 1-8). IEEE.

[14] Ruj S, Nayak A. A decentralized security framework for data aggregation and access control in smart grids. IEEE transactions on smart grid. 2013 Mar; 4(1):196-205.

[15] Khan RH, Khan JY. A comprehensive review of the application characteristics and traffic requirements of a smart grid communications network. Computer Networks. 2013 Feb 26; 57 (3):825-45.

[16] Kuzlu M, Pipattanasomporn M. Assessment of communication technologies and network requirements for different smart grid applications. InInnovative Smart Grid Technologies (ISGT), 2013 IEEE PES 2013 Feb 24 (pp. 1-6). IEEE.

[17] Lv P, Wang X, Yang Y, Xu M. Network virtualization for smart grid communications. IEEE Systems Journal. 2014 Jun; $8(2): 471-82$.

[18] Meng W, Ma R, Chen HH. Smart grid neighborhood area networks: a survey. IEEE Network. 2014 Jan; 28 (1):24-32.

[19] Kalalas C, Thrybom L, Alonso-Zarate J. Cellular communications for smart grid neighborhood area networks: A survey. IEEE Access. 2016; 4: 1469-93.

[20] Yu R, Zhang Y, Gjessing S, Yuen C, Xie S, Guizani M. Cognitive radio based hierarchical communications infrastructure for smart grid. IEEE network. 2011 Sep; 25 (5).

[21] Wu FF, Moslehi K, Bose A. Power system control centers: Past, present, and future. Proceedings of the IEEE. 2005 Nov; 93(11):1890-908.
[22] Xie X, Zhang S, Xiao J, Wu J, Pu Y. Small signal stability assessment with online eigenvalue identification based on wide-area measurement system. In Transmission and Distribution Conference and Exhibition: Asia and Pacific, 2005 IEEE/PES 2005 (pp. 1-5). IEEE.

[23] Goldsmith MW, Horne K, Hanna J, Simons R, Hamilton K. Prepared for the Conn SMART. Marc Goldsmith \& Associates LLC, May 2011.

[24] Xu S, Qian Y, Hu RQ. On reliability of smart grid neighborhood area networks. IEEE Access. 2015; 3: 2352-65.

[25] Gungor VC, Sahin D, Kocak T, Ergut S, Buccella C, Cecati C, Hancke GP. A survey on smart grid potential applications and communication requirements. IEEE Transactions on industrial informatics. 2013 Feb; 9 (1):28-42.

[26] Khan F, Ur Rehman A, Arif M, Aftab M, Jadoon BK. A survey of communication technologies for smart grid connectivity. InComputing, Electronic and Electrical Engineering (ICE Cube), 2016 International Conference on 2016 Apr 11 (pp. 256-261). IEEE.

[27] Sharma K, Saini LM. Power-line communications for smart grid: Progress, challenges, opportunities and status. Renewable and Sustainable Energy Reviews. 2017 Jan 1; 67: 704-51.

[28] Kennedy M. Leveraging investment in fiber optic communications. IEEE Smart Grid, June. 2011.

[29] Asbery CW, Jiao X, Liao Y. Implementation guidance of smart grid communication. In North American Power Symposium (NAPS), 20162016 Sep 18 (pp. 1-6). IEEE.

[30] Mahmood A, Javaid N, Razzaq S. A review of wireless communications for smart grid. Renewable and sustainable energy reviews. 2015 Jan 1; 41: 248-60.

[31] Vardakas JS, Zorba N, Verikoukis CV. A survey on demand response programs in smart grids: Pricing methods and optimization algorithms. IEEE Communications Surveys \& Tutorials. 2015 Mar; 17(1):152-78. 